\title{
Differential Effects of Long-Term Potentiation Evoked at the CA3-CA1 Synapse before, during, and after the Acquisition of Classical Eyeblink Conditioning in Behaving Mice
}

\author{
Noelia Madroñal, José M. Delgado-García, and Agnès Gruart \\ División de Neurociencias, Universidad Pablo de Olavide, 41013 Sevilla, Spain
}

\begin{abstract}
Experimentally induced long-term potentiation (LTP) is a persistent increase in synaptic strength that decays across time. In contrast, changes in synaptic strength during actual learning are gradual processes that increase with training. We have studied here the effects of LTP evoked before, during, and after the acquisition of a well known associative learning paradigm: the classical eyeblink conditioning. We used a trace paradigm, with a tone as the conditioned stimulus (CS) and an electric shock presented to the supraorbital nerve as the unconditioned stimulus (US). A single electrical pulse was presented to the Schaffer collateral-commissural pathway to evoke field EPSPs (fEPSPs) during the CS-US interval. LTP induced by high-frequency stimulation of the Schaffer collaterals lasted for 6-10 d. When LTP was evoked before conditioning, animals were unable to acquire conditioned eyeblinks if the training started $8 \mathrm{~d}$ after LTP disappearance, and no change was detected in fEPSP evoked at the CA3-CA1 synapse during conditioning. In contrast, LTP-induced animals learned as did controls when the conditioning test was presented $20 \mathrm{~d}$ after LTP had decayed to baseline, and presented a normal increase in fEPSP slopes across conditioning. When evoked during the first two conditioning sessions, LTP prevented both eyeblink conditioning and fEPSP increase. Finally, LTP did not disrupt the normal performance of a recall test of a previously acquired eyeblink conditioning. In this latter experiment, both the LTP-induced potentiation of fEPSPs and their physiological potentiation decayed across time with a similar time constant, with no apparent effect on memory recall.
\end{abstract}

Key words: long-term potentiation; electrophysiology; associative learning; hippocampus; mice; synaptic plasticity

\section{Introduction}

Electrophysiological properties and molecular events associated either with LTP have been characterized in detail, mostly by in vitro studies (Bliss and Collingridge, 1993; Malenka and Nicoll, 1999; Lynch, 2004). Nevertheless, there is little information on the possible interactions between experimentally evoked LTP and learning-dependent changes in synaptic weights. Previously, it has been shown that eyeblink conditioning evokes a concomitant change in strength at the hippocampal CA3-CA1 synapse in behaving mice (Gruart et al., 2006). Both eyeblink conditioning and the associated change in CA3-CA1 synaptic strength are prevented by high frequency stimulation (HFS) of the Schaffer collateral-commissural pathway, indicating that the evoked LTP occludes any subsequent learning (Gruart et al., 2006). Moreover, the change in synaptic strength evoked by the learning process is able to interfere with LTP induced subsequently by HFS of the involved neural circuits (Whitlock et al., 2006).

Both LTP and synaptic activity in learning evoke lasting changes in synaptic weights, and exhibit common properties

Received July 26, 2007; revised Sept. 11, 2007; accepted Sept. 12, 2007.

This work was supported by Spanish Ministerio de Educación y Ciencia Grants BFU2005-01024 and BFU200502512. We thank Roger Churchill for his editorial help.

Correspondence should be addressed to Dr. Agnès Gruart, División de Neurociencias, Universidad Pablo de Olavide, Carretera de Utrera, Kilómetro. 1, 41013 Sevilla, Spain. E-mail: agrumas@upo.es.

DOI:10.1523/JNEUROSCI.3397-07.2007

Copyright $\odot 2007$ Society for Neuroscience $\quad$ 0270-6474/07/2712139-08\$15.00/0 such as input specificity, associability, and other forms of cooperative interactions (Abraham, 2003; Morris et al., 2003). However, some questions regarding similarities and differences between HFS-induced LTP and activity-dependent synaptic plasticity remain unanswered. The first is whether LTP evoked in behaving animals induces transient, or permanent, effects on their learning capabilities, or whether its effects are restricted to the period of time during which fEPSP slopes are significantly different from baseline values. A previous report describes the effects of LTP evoked experimentally in behaving mice during the acquisition of an eyeblink conditioning (Gruart et al., 2006), but no information is available on the consequences of evoking LTP before and/or after the conditioning test. A second question arises from the observation that LTP is a decrementing synaptic phenomenon (Abraham, 2003), whereas synaptic potentiation increases across associative learning. Thus, although fEPSP slopes increase with training (Gruart et al., 2006, 2007; Whitlock et al., 2006), changes in fEPSP slopes evoked by HFS decay across time.

We studied here the effects of experimentally evoked LTP on eyeblink conditioning in behaving mice. We used a trace paradigm, presenting a tone as conditioned stimulus (CS) and an electrical shock to the supraorbital nerve as unconditioned stimulus (US). Conditioned responses were recorded from the electromyographic (EMG) activity of the orbicularis oculi muscle. Animals were implanted with chronic stimulating electrodes in the Schaffer collateral-commissural pathway and with a record- 
ing electrode in the ipsilateral CA1 area. We recorded fEPSPs evoked at the CA3-CA1 synapse across conditioning sessions and during the different HFS tests. With the help of these experimental procedures, we determined the effects on the acquisition of the conditioning task of LTP evoked 14 or $35 \mathrm{~d}$ before, during the first two conditioning sessions, or after the 10th conditioning session. Results indicate that HFS-induced effects on the learning process outlast changes evoked in fEPSP slopes, that the acquisition process is more affected than memory retrieval by experimentally induced LTP, and that, like LTP, fEPSP slopes decay spontaneously after conditioning.

\section{Materials and Methods}

Animals. Experiments were performed in $80 \mathrm{C} 57 \mathrm{BL} / 6$ male adult mice (3-5 months old; 25-35 g) obtained from an official supplier (University of Granada Animal House, Granada, Spain). Additional animals were used in some preliminary studies. Before surgery, animals were housed in separate cages ( $n=10$ per cage), but they were switched to individual cages after electrode implantation. Mice were kept on a $12 \mathrm{~h}$ light/dark cycle with constant ambient temperature $\left(21.5 \pm 1.5^{\circ} \mathrm{C}\right)$ and humidity $(55 \pm 8 \%)$. Food and water were available ad libitum. Experiments were performed in accordance with the guidelines of the European Union (2003/65/CE) and Spanish regulations (BOE 252/34367-91, 2005) for the use of laboratory animals in chronic recordings. All experimental protocols were also approved by the local Ethical Committee.

Surgery. Animals were anesthetized with $0.8-3 \%$ halothane (Astra Zeneca, Madrid, Spain), delivered by a mouse anesthesia mask (David Kopf Instruments, Tujunga, CA) and supplied from a calibrated Fluotec 5 (Fluotec-Ohmeda, Tewksbury, MA) vaporizer at a flow rate of 1-4 L/min oxygen. Animals were implanted with bipolar stimulating electrodes on the left supraorbital nerve (nearby its entrance into the orbit) and with bipolar recording electrodes in the left orbicularis oculi muscle (see Fig. $1 \mathrm{~A}$ ). Electrodes were made of $50 \mu \mathrm{m}$, Teflon-coated, annealed stainless-steel wire (A-M Systems, Carlsborg, WA). Electrode tips were bared of their isolating cover for $0.5 \mathrm{~mm}$ and bent as a hook to allow a stable insertion in the upper lid.

Animals were also implanted with bipolar stimulating electrodes in the right Schaffer collateral pathway of the dorsal hippocampus ( $2 \mathrm{~mm}$ lateral and $1.5 \mathrm{~mm}$ posterior to bregma, and $1-1.5 \mathrm{~mm}$ from the brain surface) (Paxinos and Franklin, 2001) and with a recording electrode aimed at the right CA1 stratum radiatum $(1.2 \mathrm{~mm}$ lateral and $2.2 \mathrm{~mm}$ posterior to bregma, and 1-1.5 from the brain surface). Hippocampal electrodes were made of $50 \mu \mathrm{m}$, Teflon-coated tungsten wire (Advent Research Materials, Eynsham, UK). The final location of the recording electrode in the CA1 area was determined following the field potential depth profile evoked by paired ( $20-50 \mathrm{~ms}$ of interval) pulses presented to the Schaffer collateral pathway (see below). A bare silver wire was affixed to the skull as ground. The eight wires were soldered to two four-pin sockets (RS Amidata, Madrid, Spain) which were then fixed to the skull with dental cement (Domínguez-del-Toro et al., 2004; Gruart et al., 2006).

Trace classical eyeblink conditioning. Recording sessions were performed with three animals at a time. Animals were placed in separate small $(5 \times 5 \times 10 \mathrm{~cm})$ plastic chambers located inside a larger Faraday box $(30 \times 30 \times 20 \mathrm{~cm})$. Classical conditioning was achieved using a trace paradigm (see Fig. $1 B$ ). For this, a tone $(20 \mathrm{~ms}, 2.4 \mathrm{kHz}, 85 \mathrm{~dB}$ ) was presented as a CS. The US consisted of a $500 \mu$ s, three times threshold, square, cathodal pulse applied to the supraorbital nerve. The US started $500 \mathrm{~ms}$ after the end of the CS. A total of two habituation and 10 conditioning sessions were performed for each animal. A conditioning session consisted of $60 \mathrm{CS}-\mathrm{US}$ presentations, and lasted $\approx 30 \mathrm{~min}$. For a proper analysis of the conditioned response, the CS was presented alone in $10 \%$ of the cases. CS-US presentations were separated at random by $30 \pm 5 \mathrm{~s}$. For habituation sessions, only the CS was presented, also for 60 times per session, at intervals of $30 \pm 5 \mathrm{~s}$. For recall (i.e., retrieval), the selected animals underwent two additional conditioning sessions $12 \mathrm{~d}$ after the 10 th one. As criteria, we considered a "conditioned response" the presence, during the CS-US interval, of EMG activity lasting $>10 \mathrm{~ms}$ and initiated $>50 \mathrm{~ms}$ after CS onset. In addition, the integrated EMG activity recorded during the CS-US interval had to be at least 2.5 times greater than the averaged activity recorded immediately before CS presentation (Porras-García et al., 2005).

Recording and stimulation procedures. The EMG activity of the orbicularis oculi muscle was recorded with Grass P511 differential amplifiers (Grass-Telefactor, West Warwick, RI) at a bandwidth of $0.1 \mathrm{~Hz}$ to 10 $\mathrm{kHz}$. Field EPSP recordings were also made with Grass P511 differential amplifiers through a high-impedance probe $\left(2 \times 10^{12} \Omega, 10 \mathrm{pF}\right)$. Synaptic field potentials in the CA1 area were evoked during habituation and conditioning sessions by a single $100 \mu$ s, square, biphasic (negativepositive) pulse applied to Schaffer collaterals $300 \mathrm{~ms}$ after CS presentation. Stimulus intensities ranged from 50 to $350 \mu \mathrm{A}$. For each animal, the stimulus intensity was set at $30-40 \%$ of the intensity necessary for evoking a maximum fEPSP response, (i.e., well below the threshold for evoking a population spike) (Gureviciene et al., 2004; Gruart et al., 2006). An additional criterion for selecting stimulus intensity was that a second stimulus, presented $40 \mathrm{~ms}$ after a conditioning pulse, evoked a larger $(>20 \%)$ synaptic field potential than the first (Bliss and GardnerMedwin, 1973). Field EPSP baseline values (Figs. 2, 3) were collected 15 min before LTP induction. For LTP induction, each animal was presented with an HFS consisting of five $200 \mathrm{~Hz}, 100 \mathrm{~ms}$ trains of pulses at a rate of 1 per s. This protocol was presented six times, at intervals of $1 \mathrm{~min}$. The $100 \mu$ s, square, biphasic pulses used to evoke LTP were applied at the same intensity used for the single pulse presented after CS presentation. The HFS protocol used here (combining low intensity values and a total of 600 electric shocks) allowed us to evoke LTP lasting $>5 \mathrm{~d}$, without the appearance of abnormal spikes in EEG recordings and/or over epileptic seizures (for details, see Gruart et al., 2006).

Experimental groups. For the sake of homogeneity, all experimental groups consisted of 10 successful animals. (1) The HFS-1 group received two HFS sessions 12 and $11 \mathrm{~d}$ before conditioning (see Fig. 2). The aim was to start conditioning sessions $\approx 10 \mathrm{~d}$ after HFS-evoked fEPSP changes returned to baseline values. (2) The HFS-2 group received two HFS sessions 33 and $32 \mathrm{~d}$ before conditioning (Fig. 3). The aim in this case was to start conditioning sessions $\approx 20 \mathrm{~d}$ after HFS-evoked fEPSP changes returned to baseline values. (3) The HFS-3 group received two HFS sessions before the first two conditioning sessions (Fig. 4). (4) The HFS- 4 group received two HFS sessions 1 and $2 \mathrm{~d}$ after the 10th conditioning sessions. Each HFS group was accompanied by a control group (1-4) submitted to the same experimental design, but in the absence of any HFS session. Another 35 animals were used for preliminary trials, to fix the recording procedures and checking the stability of the stimulation and recording systems.

Histology. At the end of the recording sessions, mice were deeply reanesthetized (sodium pentobarbital, $50 \mathrm{mg} / \mathrm{kg}$ ), and perfused transcardially with saline and $4 \%$ phosphate-buffered paraformaldehyde. Brains were dissected out, postfixed overnight at $4^{\circ} \mathrm{C}$ and stored in PBS. Sections were obtained in a microtome (Leica, Wetzlar, Germany) at $50 \mu \mathrm{m}$. Selected sections including the dorsal hippocampus were mounted on gelatinized glass slides and stained using the Nissl technique with $0.1 \%$ cresyl violet, to determine the location of stimulating and recording electrodes (Fig. 1C,D).

Data collection and analysis. EMG and extracellular hippocampal activity, and 1-volt rectangular pulses corresponding to CS and US presentations, were stored digitally on a computer through an analog/digital converter (1401 Plus; CED, Cambridge, England), at a sampling frequency of $11-22 \mathrm{kHz}$ and with an amplitude resolution of 12 bits. Data were analyzed off-line for quantification of conditioned responses and fEPSP slopes with the help of commercial (Spike 2 and SIGAVG from CEDM) and home-made (Porras-García et al., 2005; Gruart et al., 2006) representation programs. The slope of evoked fEPSPs was represented as the first derivative (volts per second) of fEPSP recordings (volts). For this, five successive fEPSPs were averaged, and the mean value of the slope during the rise time period (i.e., the period of the slope between the initial $10 \%$ and the final $10 \%$ of the fEPSP) was determined. Computed results were processed for statistical analysis using the SigmaStat for Windows package. Data are represented as the mean \pm SEM. Acquired data were analyzed using a two-way ANOVA test, with days as repeated 
A

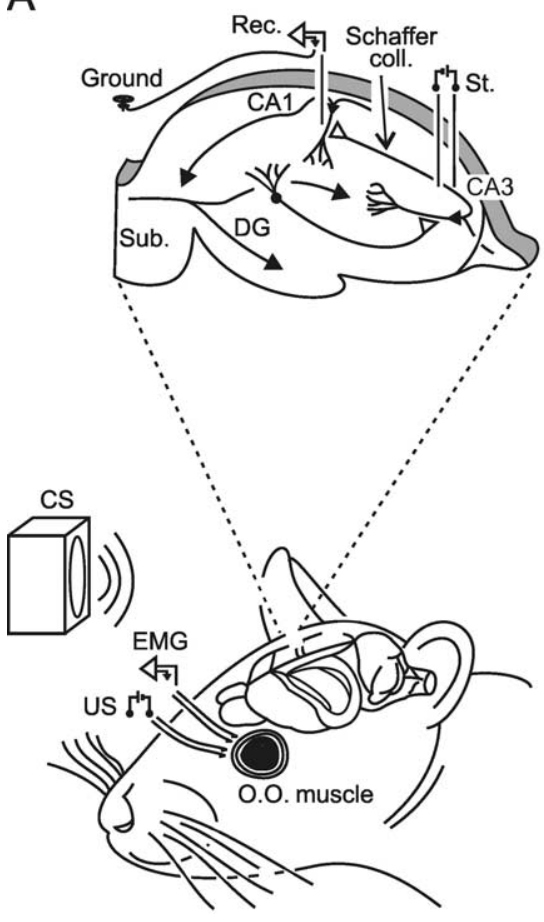

B

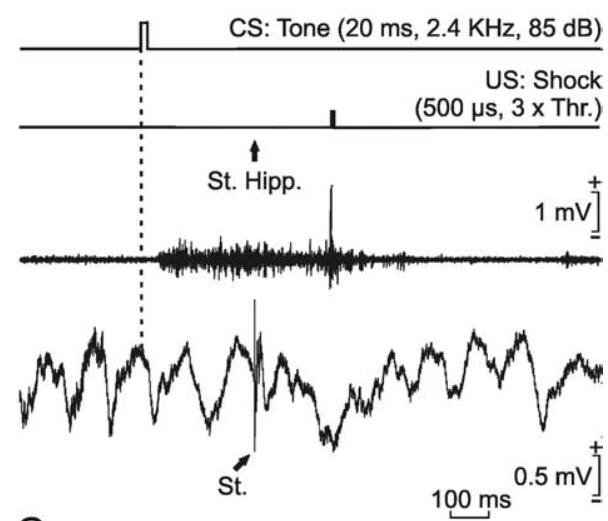

C

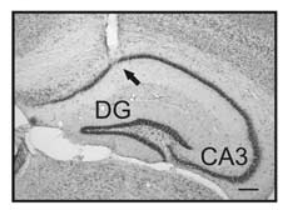

Figure 1. Experimental design. $\boldsymbol{A}$, For classical eyeblink conditioning, we used a tone as a CS. The loudspeaker was located 30 $\mathrm{cm}$ from the animal's head. Animals were implanted with bipolar stimulating electrodes on the left supraorbital nerve for US presentations. Eyelid conditioned responses were recorded with EMG electrodes implanted in the ipsilateral orbicularis oculi $(0.0$. muscle. As illustrated in the top diagram, animals were also implanted with stimulating (St.) and recording (Rec.) electrodes to activate afferents and to record fEPSPs from CA3-CA1 synapses of the right (contralateral) hippocampus. $\boldsymbol{B}$, Schematic representation of the conditioning paradigm, including CS and US stimuli, and the moment at which a single pulse was presented to Schaffer collaterals (St. Hipp.). The two bottom traces illustrate an EMG recording and an extracellular recording of hippocampal activity. Both traces were collected from the ninth conditioning session of a control animal. $\boldsymbol{C}, \boldsymbol{D}$, Photomicrographs illustrating the location of recording $(\boldsymbol{C})$ and stimulating $(\boldsymbol{D})$ electrodes. Scale bars: $200 \mu \mathrm{m}$. DG, Dentate gyrus; Sub., subiculum; D, dorsal; L, lateral; M, medial; V, ventral.

measure. Contrast analysis was added to further study significant differences.

\section{Results}

\section{EMG and fEPSP recordings in behaving mice}

The experimental design used here allows the simultaneous recording of conditioned eyeblinks and of field synaptic potentials evoked at the hippocampal CA3-CA1 synapse (Fig. 1A) (Gruart et al., 2006). In a preliminary series of experiments, we checked the stability of the in vivo recording system. In coincidence with previous reports (Gruart et al., 2006; Sahún et al., 2007), stimulating and recording electrodes implanted in the upper lid did not disturb its normal kinematics, and allowed the generation of spontaneous and electrically evoked eyeblinks. As illustrated in Figure $1 B$, conditioned eyeblinks were easily distinguished in EMG recordings, and were quantified following criteria described previously (Porras-García et al., 2005; Gruart et al., 2006).

In parallel with the acquisition of conditioned responses, we recorded the evolution of CA3-CA1 field synaptic potentials. The electrical stimulation of Schaffer collaterals in the CS-US interval evoked a definite fEPSP in the CA1 area (Fig. $1 B$ ). Recording electrodes were preferentially aimed at CA1 apical dendrites (Figs. 2-5), but, on occasion, the recorded waves presented a positive profile, corresponding to a more dorsal location (near the pyramidal cell layer) of the electrode (Schwartzkroin, 1986). The actual location of hippocampal stimulating and recording electrodes is illustrated in Figure 1, $C$ and $D$. Although the stim-

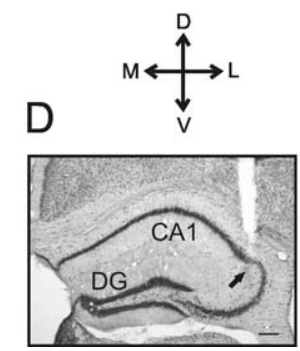

ulus applied to Schaffer collaterals disrupted the ongoing theta rhythm recorded at the $\mathrm{CAl}$ area, the rhythm reappeared in phase $100-500 \mathrm{~ms}$ later (Fig. 1 B).

Before the experiments, we checked the stability of the hippocampal recording system (de Jonge and Racine, 1985). It has been shown previously (Gruart et al., 2006) that the slope of fEPSPs evoked in the $\mathrm{CA} 1$ area by single pulses presented at the ipsilateral Schaffer collaterals (up to 60 times per day with a minimum interval of $\approx 30$ s) remains stable in the absence of any other experimental manipulation $(<10 \%$ of random variability). In this case, we checked the stability of the recording system for $50 \mathrm{~d}$, and found no statistically significant tendency toward an increase or decrease in fEPSP slopes $(\leq 12.4 \% ; n=10$; $p=0.65)$.

\section{Effects on the acquisition of conditioned eyeblinks and of CA3-CA1 field synaptic potentials of LTP induction before training} In a first series of experiments, we determined the effects of inducing LTP in behaving mice 12 (Fig. 2) and 33 (Fig. 3) d before conditioning. To obtain an fEPSP baseline, animals were stimulated at Schaffer collaterals every $5 \mathrm{~s}$ for $15 \mathrm{~min}$. The stimulus consisted of a $100 \mu$ s square, biphasic pulse. Pulse intensity was set at $30-40 \%$ of the amount necessary to evoke a maximum fEPSP response $(50-350 \mu \mathrm{A})$. LTP was evoked by the HFS protocol described in Materials and Methods. This HFS protocol was presented for 2 successive days (Figs. 2-3). To avoid evoking large population spikes and/or the appearance of abnormal EEG activities, the stimulus intensity during HFS was set at the same value used for generating baseline recordings $(<$ $350 \mu \mathrm{A})$. In this way we were able to evoke LTP affecting the whole learning process, without evoking overt seizures (Gruart et al., 2006). After HFS, the same single stimulus was presented every $5 \mathrm{~s}$ for $15 \mathrm{~min}$ on the indicated days (Figs. 2-3).

As illustrated in Figure 2A, HFS applied during two d in the HFS-1 group evoked a definite LTP that remained above baseline values for $9 \mathrm{~d}\left(F_{(11,99)}=2.998 ; p<0.002\right)$. When compared with fEPSPs evoked in the Control-1 group, fEPSP slopes recorded from the HFS-1 group were significantly larger for the first $6 \mathrm{~d}$ (Fig. $2 A)\left(F_{(11,99)}=3.04 ; p<0.002\right)$. Interestingly, by the 12th day after the first HFS session, there was no significant difference between fEPSP slopes recorded from control-1 and HFS- 1 groups. Nevertheless, and as shown in Figure $2 B$, animals included in the HFS- 1 group were unable to present a normal learning curve, reaching a plateau of $\approx 45 \%$ of conditioned responses by the eighth to the 10th conditioning sessions. In contrast, the control-1 group reached $>70 \%$ of conditioned responses from the seventh session on. The percentage of conditioned responses obtained in the control-1 group was significantly larger than the corresponding values from the HFS-1 group from the third to the 10 th conditioning sessions (Fig. $2 B)\left(F_{(11,99)}=5.235 ; p<0.001\right)$.

The slope of fEPSPs evoked in the control-1 group by single 
A
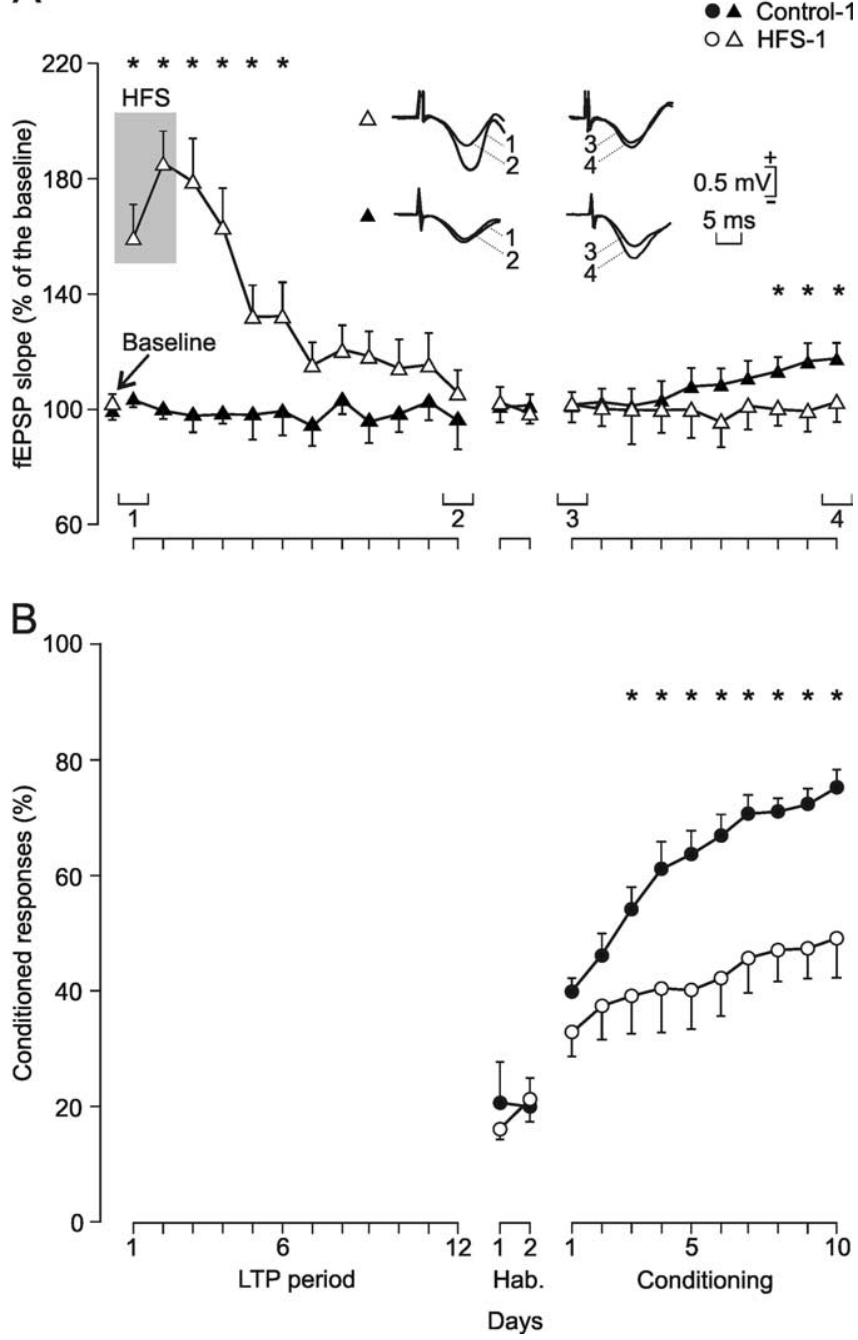

Figure 2. Evolution of $C A 3-C A 1$ synaptic field potentials and learning curves for control-1 and HFS- 1 groups. $\boldsymbol{A}, \boldsymbol{B}$, fEPSP slope ( $\boldsymbol{A}$, white triangles) and percentage of eyelid conditioned responses ( $\boldsymbol{B}$, white circles) for animals receiving HFS 12 and $11 \mathrm{~d}$ before the first habituation session (HFS-1 group). For comparison, data ( $\boldsymbol{A}$, fEPSP, black triangles; $\boldsymbol{B}$, percentage of conditioned responses, black circles) corresponding to the control-1 group are also illustrated. As a result of the LTP evoked by HFS, the fEPSP slope for the HFS-1 group was significantly larger during the first $6 \mathrm{~d}$ than that for the control-1 group $\left(\boldsymbol{A}, F_{(11,99)}=3.04 ; p<0.002\right)$. The acquisition curve presented by the HFS-1 group was also significantly different from controls ( $\boldsymbol{B}$, $\left.F_{(11,99)}=5.235 ; p<0.001\right)$. Note that during conditioning sessions there was a progressive increase in fEPSP slopes for the control-1 group, but not for the HFS-1 group. Differences in fEPSP slopes between HFS- 1 and control-1 groups were statistically significant from the eighth to the 10th conditioning sessions $\left(\boldsymbol{A}, \boldsymbol{F}_{(11,99)}=3.04 ; p<0.002\right)$. Representative fEPSPs illustrated in $\boldsymbol{A}$ were collected from the $\operatorname{LTP}(1,2)$ and conditioning $(3,4)$ periods.

electrical stimuli presented to the Schaffer collaterals increased steadily across conditioning sessions, reaching $\approx 120 \%$ of baseline values by the ninth and 10th conditioning sessions. fEPSP slopes collected from control-1 animals during conditioning were significantly larger than baseline values from the sixth to the 10 th conditioning sessions $\left(F_{(11,99)}=0.715 ; p<0.001\right)$. In contrast, fEPSPs recorded from HFS- 1 during the 10 conditioning sessions were not significantly different from baseline values ( $p=0.627)$. Finally, the differences in fEPSP slope between HFS- 1 and control- 1 groups were statistically significant from the eighth to the 10th conditioning sessions (Fig. $2 A)\left(F_{(11,99)}=3.04\right.$; $p<0.002$ ). Thus, LTP induction $12 \mathrm{~d}$ before conditioning ( $14 \mathrm{~d}$ before if habituation sessions are included) was able to prevent
A

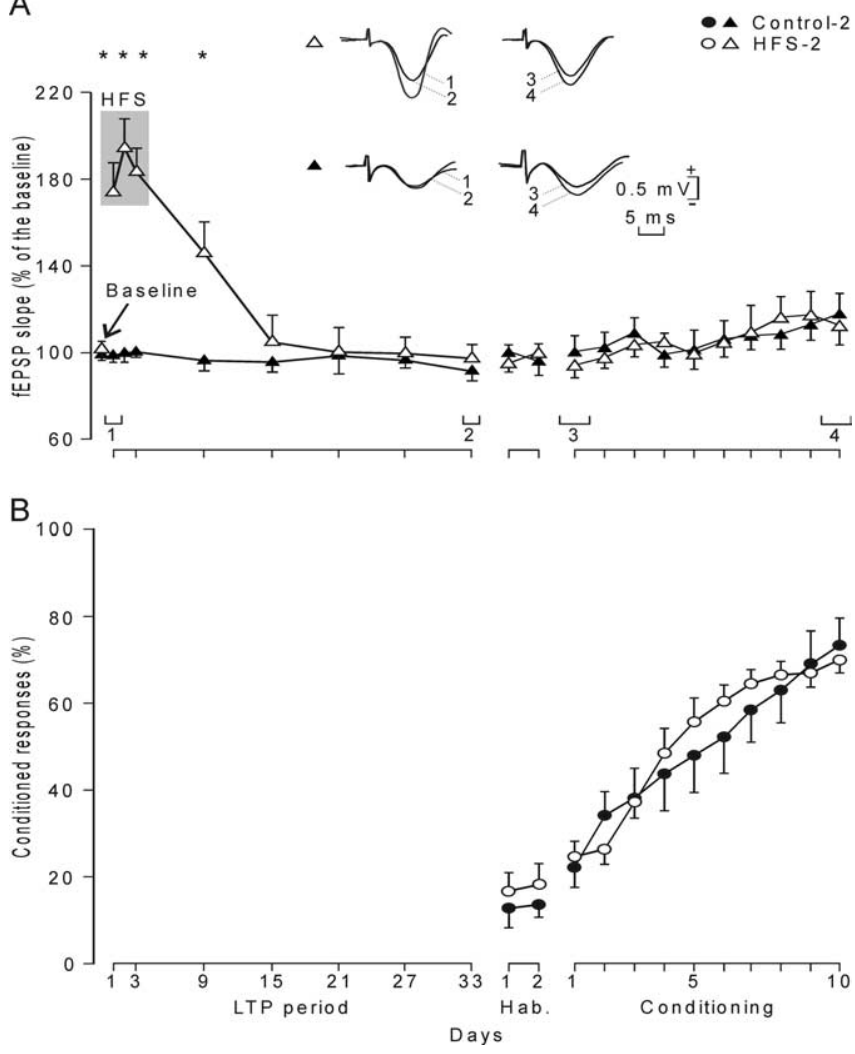

Figure 3. Evolution of $C A 3-C A 1$ synaptic field potentials and learning curves for control-2 and HFS-2 groups. $A, B$, fEPSP slope ( $\boldsymbol{A}$, white triangles) and percentage of eyelid conditioned responses ( $\boldsymbol{B}$, white circles) for animals receiving HFS 33 and $32 \mathrm{~d}$ before the first habituation session (HFS-2 group). For comparison, data ( $\boldsymbol{A}$, fEPSP, black triangles; $\boldsymbol{B}$, percentage of conditioned responses, black circles) corresponding to the control-2 group are also illustrated. As a result of the LTP evoked by HFS, the fEPSP slope for the HFS-2 group was significantly larger during the first $9 \mathrm{~d}$ than that for the control-2 group $\left(A ; F_{(7,63)}=9.683 ; p<0.001\right)$. Both groups were able to acquire the associative task, and the acquisition curves presented by the HFS-2 group were not significantly different from controls $\left(\boldsymbol{B} ; F_{(11,99)}=1.212 ; p=0.289\right)$. Note that during conditioning sessions there was a progressive increase in fEPSP slopes collected from the two groups. No significant differences in fEPSP slopes were observed between HFS-2 and control-2 groups across conditioning sessions $\left(\boldsymbol{A} ; \boldsymbol{F}_{(11,99)}=0.492 ; p=0.904\right)$. Representative fEPSPs illustrated in $A$ were collected from the LTP $(1,2)$ and conditioning $(3,4)$ periods.

the normal acquisition of a classical conditioning task, even if the evoked potentiation (as determined by the significant increase in fEPSP slope) had already disappeared.

These results prompted us to repeat the same experiment, but increasing the time period (by $>20 \mathrm{~d}$ ) between LTP induction and the beginning of classical eyeblink conditioning. In this case, the HFS protocol applied for $2 \mathrm{~d}$ to the HFS-2 group evoked an LTP that remained over baseline values for (at least) $9 \mathrm{~d}\left(F_{(7,63)}=\right.$ 15.377; $p<0.001)$. When compared with fEPSP slopes recorded in the control-2 group, those in the HFS-2 group were significantly larger for a similar period of time (Fig. $3 A)\left(F_{(7,63)}=9.683\right.$; $p<0.001)$. The slope of fEPSP recordings collected from HFS-2 and control-2 animals $15-33 \mathrm{~d}$ after the first HFS session presented no significant differences $(p=0.351)$, indicating that no LTP was detectable with our recording procedures. In this case, and as shown in Figure $3 B$, animals included in the HFS-2 group were able to present a normal learning curve, reaching percentages of conditioned responses across training similar to the corresponding values in the control-2 group (Fig. $3 B)\left(F_{(11,99)}=\right.$ $1.212 ; p=0.289)$. The percentage of conditioned responses reached by the two groups (control-2 and HFS-2) was signifi- 
A

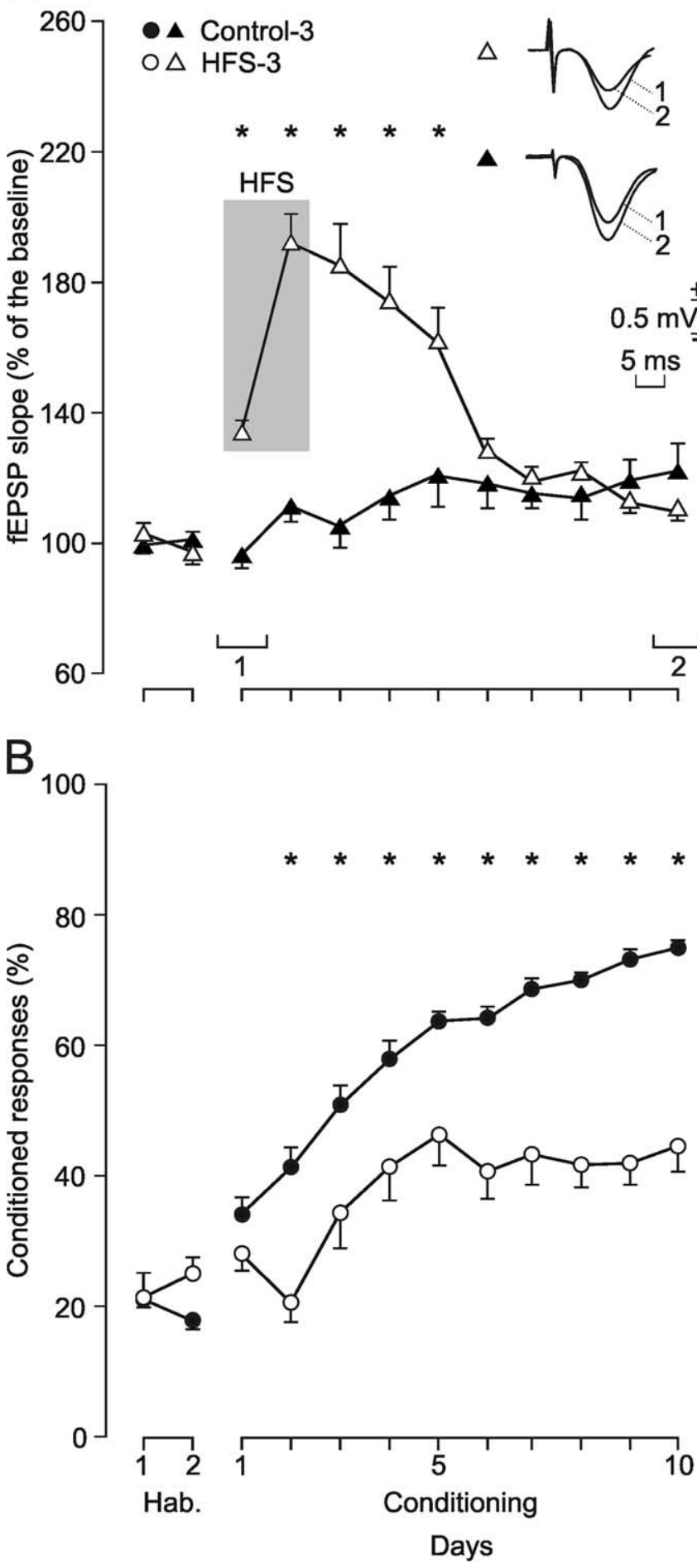

Figure 4. Evolution of CA3-CA1 synaptic field potentials and learning curves for Control-3 and HFS-3 groups. $\boldsymbol{A}, \boldsymbol{B}$, fEPSP slope ( $\boldsymbol{A}$, white triangles) and percentage of eyelid conditioned responses ( $\boldsymbol{B}$, white circles) for animals receiving HFS $30 \mathrm{~min}$ before the first two conditioning sessions (HFS-3 group). For comparison, data ( $\boldsymbol{A}$, fEPSP, black triangles; $\boldsymbol{B}$, percentage of conditioned responses, black circles) corresponding to the control-3 group are also illustrated. As a result of the LTP evoked by HFS, the fEPSP slope for the HFS-3 group was significantly larger during the first $5 \mathrm{~d}$ than that for the control-3 group $\left(\boldsymbol{A} ; F_{(11,99)}=26.517 ; p<0.001\right)$. The acquisition curve presented by the HFS-3 group was also significantly lower than controls ( $\boldsymbol{B}$, $\left.F_{(11,99)}=12.643 ; p<0.001\right)$. Note that during conditioning sessions there was a progressive increase in fEPSP slopes from the control-3 group, but not in those from the HFS-3 group. Representative fEPSPs illustrated in $\boldsymbol{A}$ were collected from the first (1) and the 10th (2) conditioning sessions.
A
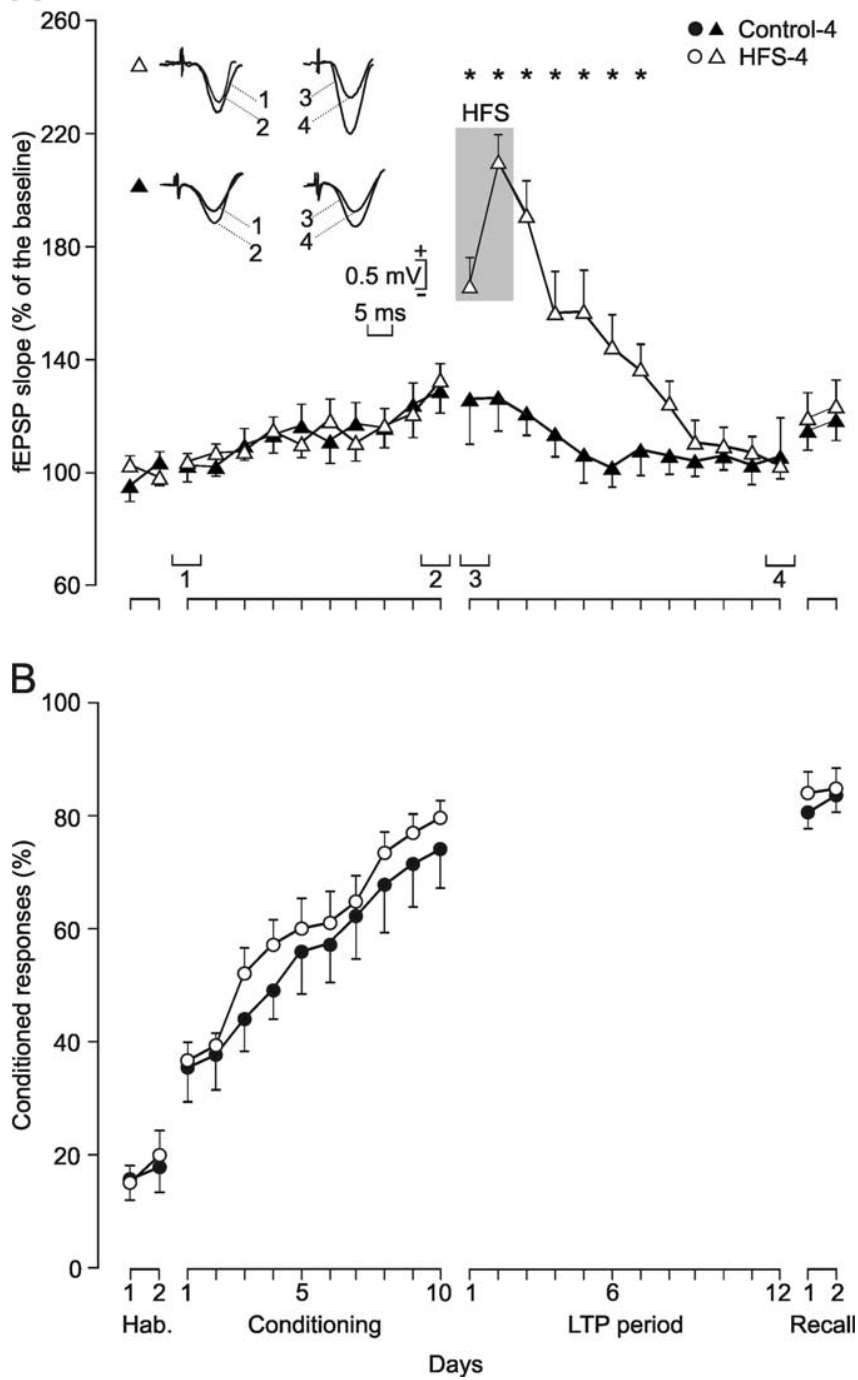

Figure 5. Evolution of $\mathrm{CA} 3-\mathrm{CA} 1$ synaptic field potentials and learning curves for control-4 and HFS-4 groups. $\boldsymbol{A}, \boldsymbol{B}$, fEPSP slope ( $\boldsymbol{A}$, white triangles) and percentage of eyelid conditioned responses during conditioning and recall periods ( $\boldsymbol{B}$, white circles) for animals receiving $\mathrm{HFS}$ the first $2 \mathrm{~d}$ after the 10 th conditioning session (HFS- 4 group). For comparison, data ( $\boldsymbol{A}$, fEPSP, black triangles; $\boldsymbol{B}$, percentage of conditioned responses, black circles) corresponding to the control-4 group are also illustrated. As a result of the LTP evoked by HFS, the fEPSP slope for the HFS-4 group was significantly larger during the first $7 \mathrm{~d}$ than that for the control-4 $\operatorname{group}\left(A ; F_{(11,99)}=\right.$ $9.442 ; p<0.001)$. Both the acquisition and recall curves presented by the HFS-4 group were equal to values collected from controls $\left(\boldsymbol{B} ; F_{(13,117)}=0.234 ; p<0.997\right)$. Note that during conditioning sessions there was a progressive increase in fEPSP slopes for the two groups (control-4 and HFS-4). Representative fEPSPs illustrated in $A$ were collected from the conditioning $(1,2)$ and LTP $(3,4)$ periods.

cantly larger than values collected during the second habituation session from the third to the 10th conditioning sessions (Fig. 3B) $\left(F_{(11,99)}=67.318 ; p<0.001\right)$.

The slope of fEPSPs evoked in both control-2 and HFS-2 groups by single electrical stimuli presented to the Schaffer collaterals increased steadily across conditioning sessions, reaching almost $120 \%$ of baseline values from the eighth to the 10 th conditioning sessions $\left(F_{(11,99)}=0.812 ; p<0.01\right)$. Differences in fEPSP slopes between HFS-2 and control-2 groups were not statistically different for any of the conditioning sessions $(p=$ $0.904)$.

In conclusion, LTP induction $33 \mathrm{~d}$ before conditioning ( $35 \mathrm{~d}$ 
before if habituation session are included) was unable to prevent the normal acquisition of a classical conditioning task.

\section{Effects on the acquisition of conditioned eyeblinks and of CA3-CA1 field synaptic potentials of LTP induction during training}

For comparative purposes, we repeated here an experiment performed and published previously (Gruart et al., 2006). In this second series of experiments, the aim was to determine the effects of LTP induction. As illustrated in Figure 4A, HFS applied during the first two conditioning sessions in the HFS-3 group evoked an LTP that remained above baseline values for the first six conditioning days $\left(F_{(11,99)}=27.546 ; p<0.001\right)$. In this case, fEPSP baseline values corresponded to those collected during the two habituation sessions. When compared with fEPSP slopes recorded in the control-3 group, those in the HFS-3 group were significantly larger for the first $5 \mathrm{~d}$ (Fig. $4 A)\left(F_{(11,99)}=26.517\right.$; $p<0.001)$.

In this situation, animals included in the HFS-3 group were unable to acquire the classical conditioning paradigm at a normal rate, reaching a plateau of $\approx 40 \%$ of conditioned responses by the fourth to the 10th conditioning sessions. In contrast, the control-3 group reached $>70 \%$ of conditioned responses from the eighth to the 10th conditioning sessions $\left(F_{(11,99)}=12.643\right.$; $p<0.001)$. The percentage of conditioned responses reached in the control-3 group was significantly larger than the corresponding values in the HFS-3 group from the second to the 10th conditioning sessions (Fig. $4 B)\left(F_{(11,99)}=12.643 ; p<0.001\right)$.

The slope of fEPSPs evoked in the control-3 group by single electrical stimuli presented to the Schaffer collaterals increased across conditioning, reaching almost $118 \%$ of baseline during the fifth, sixth, ninth, and 10th conditioning sessions $\left(F_{(11,99)}=\right.$ 40.78; $p<0.01)$.

As already described, and further confirmed here, LTP induction at the beginning (first two sessions) of a classical eyeblink conditioning was able to prevent its normal acquisition, even if the evoked potentiation (as determined by the significant increase in fEPSP slope) had already disappeared (for the last four conditioning sessions).

\section{Effects on the acquisition of conditioned eyeblinks and of CA3-CA1 field synaptic potentials of LTP induction after training}

In a third series of experiments, we attempted to determine the effects of LTP induction in a group of animals (HFS-4) that had already acquired an eyeblink conditioning. As illustrated in Figure $5 B$, the two groups of animals (control-4 and HFS-4) were able to accomplish a classical conditioning test. Indeed, the percentage of conditioned responses reached by these two groups was significantly larger than values collected during the second habituation session from the third to the 10th conditioning sessions (Fig. $5 B)\left(F_{(13,117)}=82.82 ; p<0.001\right)$. There were no significant differences in the learning curves between the two groups (Fig. $5 B)\left(F_{(13,117)}=0.234 ; p<0.997\right)$.

The slope of fEPSPs evoked in both Control-4 and HFS-4 groups by single pulses presented to the Schaffer collaterals increased steadily across conditioning sessions, reaching $118 \%$ of baseline values from the fifth to the 10th conditioning sessions $\left(F_{(11,99)}=6.506 ; p<0.001\right)$. Differences in fEPSP slopes between HFS-4 and control-4 groups were not statistically significant for any of the conditioning sessions ( $p=0.763$ ).

In this series of experiments, the HFS protocol applied for $2 \mathrm{~d}$ to the HFS-4 group evoked an LTP that remained over baseline values for the first $8 \mathrm{~d}$ of the LTP period $\left(F_{(11,99)}=10.487 ; p<\right.$ $0.001)$. In this case, fEPSP baseline values corresponded to those collected during the two habituation sessions. When compared with fEPSP slopes recorded in the control-4 group, those in the HFS-4 group were significantly larger for the first $7 \mathrm{~d}$ of the LTP period (Fig. $5 A)\left(F_{(11,99)}=9.442 ; p<0.001\right)$. Interestingly, and in the absence of any additional training, the slope of fEPSPs recorded in control-4 animals decreased spontaneously from values reached during the 10th conditioning session to baseline values in $4 \mathrm{~d}$. Thus, fEPSP values recorded in control- 4 animals from the fifth to the 10th day after the last conditioning session (i.e., during the LTP period) were equal to those collected during the second habituation session ( $p=0.563$ ). Finally, fEPSPs recorded from the control-4 and HFS-4 groups presented similar slopes during the last $4 \mathrm{~d}$ (ninth to 12th days) of the LTP period (Fig. $5 A)$.

According to the results collected during the two recall sessions (Fig. $5 A, B$ ), both groups of animals were able to generate percentages of conditioned responses in the range of those reached during the 10th conditioning session. Thus, the control-4 group presented a mean of $74.1 \pm 6.9$ conditioned responses during the 10 th conditioning session and $83.5 \pm 2.1$ during the second recall session. The HFS group reached similar scores $(80.2 \pm 3.5$ during the 10 th conditioning session against $85.2 \pm 3.5$ during the second recall session). Those values were not significantly different for the two groups ( $p=0.997)$. At the same time, fEPSP slopes recorded from both control-4 and HFS-4 groups during the two recall sessions increased significantly $\left(F_{(13,117)}=6.543 ; p \leq 0.01\right)$ with respect to values collected during the 12th day of the LTP period, but were similar to values collected during the 10th conditioning session ( $p \geq 0.533)$.

These results indicated that LTP evoked after the acquisition of a classical eyeblink conditioning has no effect on its retrieval if the recall sessions are performed after fEPSP slopes have returned to baseline values. Another interesting finding is that the activitydependent increase in CA3-CA1 synaptic strength reached by the 10th conditioning session exhibited a passive decay during the following days, reaching baseline values by the fifth day after the 10 th conditioning session.

\section{Discussion}

We have shown here that LTP, evoked by HFS of Schaffer collaterals, disturbs the acquisition of eyeblink responses when evoked $14 \mathrm{~d}$ before conditioning or during the conditioning process, but not when evoked well before ( $35 \mathrm{~d}$ ) or after training. Evidently, LTP induction not only artificially modified the (expected) synaptic response of CA1 pyramidal cells to Schaffer collateralcommissural pathway commands, but also blocked all of the subsequent information transfer toward the following neuronal synaptic contacts within hippocampal (and extra-hippocampal) circuits (Buzàki et al., 1988). It can therefore be proposed that the experimental disturbance of a relevant synaptic step (in this case, the CA3-CA1 synapse) is apparently enough to disturb the functional state corresponding to a given type (in this case, a trace tone-shock paradigm) of associative learning. In this context, a functional state should be considered as a network of distributed and specific synaptic weights involved in a specific type of learning processes (Delgado-García and Gruart, 2002).

\section{Effects of LTP on associative learning when evoked before the conditioning test}

Hippocampal lesions and/or temporal lobe epileptic conditions in humans are able to evoke both anterograde and immediate 
retrograde amnesia, as repeatedly corroborated with different neuropsychological tests (Halgren et al., 1991; Thompson, 1991; Squire and Alvarez, 1995). In this regard, it has been shown that LTP induction in the perforant pathway is able to prevent spatial learning (McNaughton et al., 1986; Castro et al., 1989; Moser et al., 1998; Dragoi et al., 2003). Apparently, the evoked disruption of spatial memory by the induction of saturating LTP is only fully evident by the repeated stimulation of different sites across hippocampal circuits (Moser et al., 1998). It has also been reported that a distributed alteration of entorhinal and hippocampal circuits evoked by hippocampal kindling is sufficient to disrupt spatial learning (Leung and Shen, 2006). The present results further confirm that LTP evoked before training is able to prevent the acquisition of new motor abilities. In our case, we have used a trace conditioning paradigm, a well known associative learning test which requires the participation of the hippocampus (Thompson, 1988; Moyer et al., 1990; Múnera et al., 2001; Gruart et al., 2006).

Importantly, the present results also suggest that LTP's deleterious effects on associative learning are present not only during the period in which fEPSP slopes are displaced from baseline physiological values. As illustrated in Figure 2, trace eyeblink conditioning is severely disrupted even when fEPSP values have returned to baseline values $5 \mathrm{~d}$ before the first conditioning session. These results might indicate that experimentally induced LTP (in this case by HFS of Schaffer collaterals) can result in a permanent disruption of associative learning (Gruart et al., 2006) and apparently contradict one of the predictions (anterograde alteration) of the synaptic plasticity and memory hypothesis (Martin et al., 2000). Nevertheless, when a longer period of time $(>25 \mathrm{~d})$ was allowed between the end of a detectable change in synaptic strength evoked by HFS and the beginning of conditioning sessions (Fig. 3), LTP-evoked mice were able to acquire the conditioning test as controls did. Thus, although LTP does not evoke a permanent deficit in anterograde memory, its effects remain for a certain period of time (days) after fEPSP slopes have already returned to baseline values. Therefore, functional changes (evoked by either LTP or kindling) in the relative synaptic weights within hippocampal circuits (Martin et al., 2000; Morris et al., 2003; Gruart et al., 2006) and/or HFS-induced changes in transcriptional processes (Bourtchouladze et al., 1994; Davis et al., 2000; Inda et al., 2005; Gruart et al., 2007) are still able to disrupt associative learning even in the absence of detectable changes in fEPSP slopes at the stimulated CA3-CA1 synapse. In this regard, the LTP evoked here can be considered a type 2 lateLTP (Abraham, 2003) with a time constant of $\approx 3.5 \mathrm{~d}$, which is dependent on gene expression and protein synthesis (Nguyen et al., 1994).

\section{Effects of LTP on associative learning when evoked during the conditioning test}

The present results further confirm previous experiments (Gruart et al., 2006) in which LTP prevented the acquisition of a trace eyeblink conditioning when evoked at different moments of the acquisition process. They also confirm the presence of an activity-dependent increase in synaptic activity taking place at the CA3-CA1 synapse during the acquisition process. This physiological, plastic process is disrupted by the HFS-evoked saturation and/or occlusion of CA3-CA1 synaptic mechanisms (Gruart et al., 2006). The opposite phenomenon has also been reported, namely, that learning-induced synaptic potentiation is able to occlude HFS-induced LTP (Whitlock et al., 2006).

Although the discharge rate of identified hippocampal CA1 pyramidal neurons does not seem to encode the kinetic peculiarities of conditioned eyeblinks, it has been convincingly shown that CA1 firing is linearly related with the progressive acquisition of the eyelid learned response, with a gain of $\approx 0.035 \mathrm{spikes} / \mathrm{s} /$ trial, as measured in behaving cats during trace (tone-shock) classical conditioning (Múnera et al., 2001). This slow building up of the hippocampal neuronal response across conditioning is similar to the small increase in the slope of the fEPSP evoked at the apical dendrite of CA1 pyramidal cells by single pulses applied to Schaffer collaterals (i.e., a $0.03 \%$ increase in fEPSP slope/trial).

\section{Effects of LTP on associative learning when evoked after the conditioning test}

It is well known that systemic drug administration, application of electroconvulsive shocks, and the induction of LTP or afterdischarges during memory reactivation or reconsolidation, are able to evoke a noticeable amnesia of already acquired learning (Sara, 2000; Nader, 2003; Gruart et al., 2006; Leung and Shen, 2006). In this regard, it has been proposed that both retrieval and reconsolidation are able to alter memory storage, depending on synaptic plastic processes taking place at selective cortical and subcortical structures (Inda et al., 2005; Doyere et al., 2007). Specifically, it has been shown previously that LTP evoked at the CA3-CA1 synapse during recall produces a severe decrease in the percentage of acquired conditioned eyelid responses (Gruart et al., 2006). These results indicate that memories become particularly labile when recalled, and require the contribution of hippocampal circuits for their restorage. In contrast, our results clearly confirm that LTP evoked in the hippocampal circuit following trace eyeblink conditioning has no additional effect on a recall or rehearsal of the acquired association if the LTP has already returned to fEPSP baseline values. A comparison of results shown in Figures 2 and 5 also suggests that the hippocampus is not required after memory consolidation (Squire and Alvarez, 1995; Morris et al., 2003), at least in the same way that it is required for the acquisition of associative learning (Gruart et al., 2006). However, these results are somewhere paradoxical, because hippocampal circuits are able to store persistent (time constant $>25 \mathrm{~d}$ ) LTP [denominated LTP3 by Abraham (2003)], but do not seem to be involved in long-term memory. Theoretical models of neural networks related to the acquisition of sequential tasks indicate that it is possible to modify the synaptic weight of individual synapses without losing memories stored previously in the network (Robins and Frean, 1998). This would explain why hippocampal circuits deal better with consolidated memories than with the acquisition of new associative learning tasks.

\section{A comparison between LTP and activity-dependent changes in synaptic strength}

It has been demonstrated previously that LTP experimentally evoked at the CA3-CA1 synapse is able to occlude further changes in strength of this synaptic site, and consequently to impair trace eyeblink conditioning (Gruart et al., 2006). The reverse is also apparently true, namely, that learning-induced synaptic potentiation occluded HFS-induced LTP (Whitlock et al., 2006). Associative learning and experimentally induced LTP share some similarities, such as input specificity, associability, activity-dependent changes in synaptic weights, and common presynaptic and postsynaptic processes (Bliss and GardnerMedwin, 1973; Bliss and Lømo, 1973; Bliss and Collingridge, 1993; Bolshakov et al., 1997; Malenka and Nicoll, 1999; Abraham, 2003; Morris et al., 2003; Nicoll, 2003; Bliss et al., 2006). Nevertheless, there are important differences between the two phe- 
nomena. For example, activity-dependent synaptic potentiation is a rather slow process that increases with training, at the least for classical eyeblink conditioning (Gruart et al., 2006), whereas LTP is rapidly induced and decays exponentially (with different time constants) across time (Abraham, 2003). Interestingly, and as illustrated in Figure $5 A$, the synaptic potentiation evoked during training decays (with a time constant of 2-3 d) to that of the HFS-induced LTP after the last conditioning session. Because memories (experimentally evoked or induced by the conditioning test) survive any further detectable change in synaptic strength, we have to assume that LTP shares important subcellular and molecular mechanisms (Bliss and Collingridge, 1993; Nguyen et al., 1994; Gruart et al., 2007) and/or neural network properties (Robins and Frean, 1998; Abraham, 2003) with the physiological decays in synaptic potentiation described here after the end of conditioning sessions. A detailed study of this (common) neural process will surely be helpful in understanding information transfer from changes in synaptic weights to subcellular and/or molecular encoding of acquired knowledge.

\section{References}

Abraham WC (2003) How long will long-term potentiation last? Phil Trans R Soc Lond B Biol Sci 358:735-744.

Bliss TVP, Collingridge GL (1993) A synaptic model of memory: long-term potentiation in the hippocampus. Nature 361:31-39.

Bliss TVP, Gardner-Medwin AR (1973) Long-lasting potentiation of synaptic transmission in the dentate area of the unanaesthetized rabbit following stimulation of the perforant path. J Physiol (Lond) 232:357-374.

Bliss TVP, Lømo T (1973) Long-lasting potentiation of synaptic transmission in the dentate area of the anaesthetized rabbit following stimulation of the perforant path. J Physiol (Lond) 232:331-356.

Bliss TV, Collingridge GL, Laroche S (2006) Neuroscience. ZAP and ZIP, a story to forget. Science 313:1058-1059.

Bolshakov VY, Golan H, Kandel ER, Siegelbaum SA (1997) Recruitment of new sites of synaptic transmission during the cAMP-dependent late phase of LTP at CA3-CA1 synapses in the hippocampus. Neuron 19:635-651.

Bourtchouladze R, Frenguelli B, Blendy J, Cioffi D, Schutz G, Silva AJ (1994) Deficient long-term memory in mice with a targeted mutation of the cAMP-responsive element-binding protein. Cell 79:59-68.

Castro CA, Silbert LH, McNaughton BL, Barnes CA (1989) Recovery of spatial learning deficits after decay of electrically induced synaptic enhancement in the hippocampus. Nature 342:545-548.

Davis S, Vanhoutte P, Pagès C, Caboche J, Laroche S (2000) The MAP/ERK cascade targets both Elk-1 and cAMP response element-binding protein to control long-term potentiation-dependent gene expression in the dentate gyrus in vivo. J Neurosci 20:4563-4572.

de Jonge M, Racine RJ (1985) The effects of repeated induction of long-term potentiation in the dentate gyrus. Brain Res 328:181-185.

Delgado-García JM, Gruart A (2002) The role of interpositus nucleus in eyelid conditioned responses. Cerebellum 1:289-308.

Domínguez-del-Toro E, Rodríguez-Moreno A, Porras-García E, SánchezCampusano R, Blanchard V, Lavilla M, Böhme GA, Benavides J, DelgadoGarcía JM (2004) An in vitro and in vivo study of early deficits in associative learning in transgenic mice that over-express a mutant form of human APP associated with Alzheimer's disease. Eur J Neurosci 20:1945-1952.

Doyere V, Debiec J, Monfils MH, Schafe GE, LeDoux JE (2007) Synapsespecific reconsolidation of distinct fear memories in the lateral amygdala. Nat Neurosci 10:414-416.

Dragoi G, Harris KD, Buzsàki G (2003) Place representation within hippocampal networks is modified by long-term potentiation. Neuron 39:843-853.

Gruart A, Muñoz MD, Delgado-Garcia JM (2006) Involvement of the CA3CA1 synapse in the acquisition of associative learning in behaving mice. J Neurosci 26:1077-1087.

Gruart A, Sciarretta C, Valenzuela-Harrington M, Delgado-Garcia JM,
Minichiello L (2007) Mutation at the TrkB PLC $\gamma$-docking site affects hippocampal LTP and associative learning in conscious mice. Learn Mem 14:54-62.

Gureviciene I, Ikonen S, Gurevicius K, Sarkaki A, van Groen T, Pussinen R, Ylinen A, Tanila H (2004) Normal induction but accelerated decay of LTP in APP + PS1 transgenic mice. Neurobiol Dis 15:188-195.

Halgren E, Stapleton J, Domalski P, Swartz BE, Delgado-Escueta AV, Walsh GO, Mandelkern M, Blahd W, Ropchan J (1991) Memory dysfunction in epilepsy patients as a derangement of normal physiology. Adv Neurol 55:385-410.

Inda MC, Delgado-García JM, Carrion AM (2005) Acquisition, consolidation, reconsolidation, and extinction of eyelid conditioning responses require de novo protein synthesis. J Neurosci 25:2070-2080.

Leung LS, Shen B (2006) Hippocampal CA1 kindling but not long-term potentiation disrupts spatial memory performance. Learn Mem 13:18-26.

Lynch MA (2004) Long-term potentiation and memory. Physiol Rev 84:87-136.

Malenka R, Nicoll RA (1999) Long-term potentiation-a decade of progress? Science 285:1870-1874.

Martin SJ, Grimwood PD, Morris RGM (2000) Synaptic plasticity and memory: an evaluation of the hypothesis. Annu Rev Neurosci 23:649-711.

McNaughton BL, Barnes CA, Rao G, Baldwin J, Rasmussen M (1986) Longterm enhancement of hippocampal synaptic transmission and the acquisition of spatial information. J Neurosci 6:563-571.

Morris RGM, Moser EI, Riedel G, Martin SJ, Sandin J, Day M, O'Carroll CO (2003) Elements of a neurobiological theory of the hippocampus: the role of activity-dependent synaptic plasticity in memory. Phil Trans R Soc Lond B 358:773-786.

Moser EI, Krobert KA, Moser MB, Morris RGM (1998) Impaired spatial learning after saturation of long-term potentiation. Science 281:2038-2042.

Moyer Jr JR, Deyo RA, Disterhoft JF (1990) Hippocampectomy disrupts trace eye-blink conditioning in rabbits. Behav Neurosci 104:243-252.

Múnera A, Gruart A, Muñoz MD, Fernández-Mas R, Delgado-García JM (2001) Discharge properties of identified CA1 and CA3 hippocampus neurons during unconditioned and conditioned eyelid responses in cats. J Neurophysiol 86:2571-2582.

Nguyen PV, Abel T, Kandel ER (1994) Requirement of a critical period of transcription for induction of a late phase of LTP. Science 265:1104-1107.

Nader K (2003) Memory traces unbound. Trends Neurosci 26:65-72.

Nicoll RA (2003) Expression mechanisms underlying long-term potentiation: a postsynaptic view. Phil Trans R Soc Lond B 358:721-726.

Paxinos G, Franklin KBJ (2001) The mouse brain in stereotaxic coordinates. London: Academic

Porras-García E, Cendelin J, Domínguez-del-Toro E, Vožeh F, DelgadoGarcía JM (2005) Purkinje cell loss affects differentially the execution, acquisition and prepulse inhibition of skeletal and facial motor responses in Lurcher mice. Eur J Neurosci 21:979-988.

Robins A, Frean M (1998) Local learning algorithms for sequential tasks in neural networks. Adv Comput Intell 2:221-227.

Sahún I, Delgado-García JM, Amador-Arjona A, Giralt A, Alberch J, Dierssen M, Gruart A (2007) Dissociation between CA3-CA1 synaptic plasticity and associative learning in TgNTRK3 transgenic mice. J Neurosci 27:2253-2260.

Sara SJ (2000) Retrieval and reconsolidation: toward a neurobiology of remembering. Learn Mem 7:73-84.

Schwartzkroin PA (1986) Regulation of excitability in hippocampal neurons. In: The hippocampus (Isacson RL, Pribram KH, eds), pp 113-136. New York: Plenum.

Squire LR, Alvarez P (1995) Retrograde amnesia and memory consolidation: a neurobiological perspective. Curr Op Neurobiol 5:169-177.

Thompson PJ (1991) Memory function in patients with epilepsy. Adv Neurol 55:369-384.

Thompson RF (1988) The neural basis of basic associative learning of discrete behavioral responses. Trends Neurosci 11:152-155.

Whitlock JR, Heynen AJ, Shuler MG, Bear MF (2006) Learning induces long-term potentiation in the hippocampus. Science 313:1093-1097. 